\title{
Thermal calculations of underground oil pipelines
}

\author{
Boris Moiseev ${ }^{1}$, Yury Zemenkov ${ }^{1}$, Nikita Nalobin ${ }^{1}$, Sergey Dudin ${ }^{1, *}$ \\ ${ }^{1}$ Tyumen Industrial University, Volodarskogo str., 38, Tyumen, 625000, Russia
}

\begin{abstract}
Operation of oil pipelines in the frozen soil causes heat exchange between the pipeline and the soil and formation of a melt zone which leads to deformation of pipelines. Terms of construction and operation of oil pipelines are greatly related to their temperature conditions. In this regard it is necessary to know the laws of formation of thawing halos around oil pipelines. Thus, elucidation of laws of formation of thawing halos around oil pipelines and determination of optimal conditions for their installation during construction in areas of permafrost in the north of Tyumen region is a very urgent task. The authors developed an algorithm and a computer program for construction of the temperature field of the frozen soil. Some problems have been solved basing on the obtained dependences and graphs of the dependence were constructed. Research and calculations made on the underground oil pipeline construction allowed the authors to give recommendations aimed at increasing the reliability of oil pipelines.
\end{abstract}

\section{Introduction}

When pumping oil and petroleum products by pipeline laid in the frozen ground their temperature drops as a result of heat exchange. Reduced temperature of the oil causes an increase in the viscosity and resistance to friction.

Thermal interaction between the pipeline and the environment depends on a way of laying and a pumping method and affects the capacity of heating and pumping stations. Thermal interaction plays a significant role when pumping high-viscosity oil in a heated state. When conducting thermal calculations, the law of temperature distribution along the length of the pipeline and the heat loss are determined. These data are taken into account when determining the throughput capacity, selecting the spacing of pumping and heating stations, calculating pipelines for strength, stability and insulation thickness. The thermal calculation data are used for the operational dispatch management of pipelines, determination of a safe time to stop "hot" pumping of high-viscosity oil. Thermal conditions are essential if operating modes of the pipeline are unsteady - start, stops $[7,8,9$, $10]$.

* Corresponding author: srghome@mail.ru 
When carrying out thermal calculation it is necessary to have data on the thermal properties of the oil and insulation. These include: density, heat capacity, thermal conductivity, thermal diffusivity, viscosity. It is necessary to obtain climatological data on the pipeline area, the temperature parameters of the air and soil at the depth of the pipeline laying, solar radiation, and the thickness of snow cover. Physical and thermophysical properties are generally determined by reference tables for oils from different deposits $[7,8$, 9]. In rough calculations semi-empirical and empirical equations are used to determine the thermophysical characteristics [7, 8, 9].

The heat capacity of oil:

$$
c_{p}=\Delta^{-0,5}\left(1,687+3,39 \cdot 10^{3} t\right)
$$
${ }^{\circ} \mathrm{C}$.

where ${ }^{c_{p}}$ - the specific heat, $\mathrm{kJ} /(\mathrm{kg} \cdot \mathrm{K}) ; \Delta_{-}$the relative oil $/$water density at $\mathrm{t}=15$

The specific heat of oils from different deposits varies in the range of $1.6 \div 2.5 \mathrm{~kJ} /(\mathrm{kg}$. $\mathrm{K})$.

The coefficient of thermal conductivity of oil $\lambda$ is determined by the formula:

$$
\lambda=(0,137 / \Delta) \cdot\left(1-0,54 \cdot 10^{-3} t\right)
$$

And varies from 0.1 to $0.16 \mathrm{~W} /(\mathrm{m} \cdot \mathrm{K})$. The coefficient of thermal diffusivity is found only for transient modes - heating and cooling processes - and is defined as $a=\lambda / c \rho$.

The most common equation to calculate oil temperature changes along the length of the pipeline is the equation of V. G. Shukhov [7]:

$$
t=t_{g r}+\left(t_{1}-t_{g r}\right) \mathrm{e}^{-\frac{k \pi D}{M c_{p}} x},
$$

where $\mathrm{t}$ - the oil temperature in the considered section, ${ }^{\circ} \mathrm{C} ; \operatorname{tgr}-$ the soil temperature at the depth of the oil pipeline laying, $\mathrm{t} 1$ - the temperature of oil entering the section, ${ }^{\circ} \mathrm{C}$; $\mathrm{D}-$ the pipeline diameter, $\mathrm{m} ; \mathrm{x}-$ the distance to the section under consideration from the entrance to the section, $\mathrm{m} ; \mathrm{M}-$ the mass flow rate of oil through the pipeline, $\mathrm{kg} / \mathrm{s} ; \mathrm{cp}-$ the mean oil heat capacity, $\mathrm{J} /(\mathrm{kg} \cdot \mathrm{K}) ; \mathrm{k}$ - the heat transfer coefficient, $\mathrm{W} /(\mathrm{m} \cdot \mathrm{K})$.

However, operating data show significant deviations of the actual observed temperature at the end of the section from the rated one. This is due to the fact that the heat of the internal friction which arises when oil moves through the pipeline is not taken into account. This value is small for small diameters and expenses and it can be ignored, but for main oil pipelines of large diameters operating at full capacity it must be considered [7]. One way to limit the thermal effects of the pipeline on the frozen soil is the use of polyurethane foam insulation (PUF- insulation with a thermal conductivity coefficient $\lambda=0.036 \mathrm{~W} /(\mathrm{m} \cdot \circ \mathrm{C})$ ) [7].

\section{Research object}

To determine thawing halos it is necessary to know the temperature field in the soil around the pipeline. The existing techniques for calculating the temperature field of the soil around oil pipelines are recommended by one of the Forchheimer modifications that give error of up to $30 \%$. Experimental studies on existing pipelines are costly and lengthy. Currently, the numerical simulation is increasingly more commonly applied $[1,2,3]$. Numerical experiment compares favourably to a lab one or under production conditions so that it is possible to investigate the influence of each defining parameter of the process under study separately in a wide range of values $[4,5,6]$. To construct the temperature field of the 
frozen soil around the pipeline the authors developed an algorithm and a program for calculations $[5,6]$.

\section{Methods}

The program is written in the style of a multiple document application (MDI). The program includes a set of calculations: determination of the soil temperature on time at a depth of the pipeline; constructing a graph of the soil temperature on the depth of the pipeline; determination of the temperature dependence on the pipe surface on the depth of the pipeline; determination of the dependence of the heat flow from the pipe surface on the depth of the pipeline; determination of the heat medium temperature dependence on the distance; the dependence of the thicknesses of the insulating layer at a given temperature difference on the distance to the entrance; the dependence of the size of the thawing zone (upper and lower limit) on the depth of the pipeline at given values of the temperature difference and the thickness of the insulating layer. All the received graphs of dependences can be stored as pictures in a bitmap format, and the calculation data - in a digital format as a text file [6].

\section{Results and discussion}

In this article the authors discuss the following issues:

The fluid temperature drop along the length of the heat conductor can be found by the formula:

$$
t_{g}(x)=t_{g r}+\left(t^{\prime}-t_{g r}\right) \exp \left(-\frac{x}{l_{h a r}}\right)
$$

where:

$$
l_{\text {har }}=G C_{g} R=G C_{g}\left(\frac{\alpha}{2 \pi \lambda_{i z}}+\frac{\beta}{2 \pi \lambda_{g r}}\right)
$$

The temperature at the end point of consumption:

$$
t^{\prime \prime}(x)=t_{g}^{\prime \prime}(x)=t_{g r}+\left(t^{\prime}-t_{g r}\right) \exp \left(-\frac{L}{l_{\text {har }}}\right)
$$

where $\quad L-$ the total length of the pipeline.

The dependence of the soil temperature at the depth of the pipeline can be represented as:

$$
t_{h}=t_{n}(\tau)\left(1-\frac{h}{H_{m}(\tau)}\right)
$$

After substitution $t_{n}(\tau)$ into this expression we get:

$$
t_{h}=A \sin \left(\frac{\pi \tau}{\tau_{x}}\right)\left(1-\frac{h}{H_{m}(\tau)}\right) .
$$

In addition, the time when the temperature front is at the depth of the pipeline is determined by: 


$$
\tau_{0}=\tau_{x}\left(\frac{h}{H_{t}}\right)^{2}
$$

Thus, temperature changes at the depth of the pipeline $\mathrm{h}$ can be calculated by the following dependence. The coldest temperature at this depth is equal to the minimum of this function, and it will participate in further calculations.

$$
t_{h}=\left\{\begin{array}{c}
0 ; \tau<\tau_{0} \\
A \sin \left(\frac{\pi \tau}{\tau_{x}}\right)\left(1-\frac{h}{H_{m}(\tau)}\right) ; \tau_{0} \leq \tau \leq \tau_{x} \\
0 ; \tau<\tau_{0}
\end{array}\right.
$$

To determine the temperature on the surface of the pipeline we form a balance equation for heat flows.

The flow from the hot fluid to the surface of the pipe:

$$
q_{l}=\frac{2 \pi \lambda_{i z}\left(t_{g}-t_{t}\right)}{\ln \frac{D_{n}+2 \delta_{i z}}{D_{n}}}=\frac{2 \pi \lambda_{i z}\left(t_{g}-t_{t}\right)}{\alpha} .
$$

The flow from the hot pipe to the frozen soil:

$$
q_{l}=\frac{2 \pi \lambda_{g r}\left(t_{t}-t_{g r}\right)}{\ln \left[\frac{2 h}{D_{n}+2 \delta_{i z}}+\sqrt{\left(\frac{2 h}{D_{n}+2 \delta_{i z}}\right)^{2}-1}\right]}=\frac{2 \pi \lambda_{g r}\left(t_{t}-t_{g r}\right)}{\beta}
$$

The balance equation in this case can be written:

$$
\frac{q_{l} \alpha}{2 \pi \lambda_{i z}}+\frac{q_{l} \beta}{2 \pi \lambda_{g r}}=t_{g}-t_{g r}
$$

where the averaged thermal conductivity equal to the mean value of the frozen and thawed soil. Hence we can get the value of the quantity of flow:

$$
q_{l}=\frac{t_{g}-t_{g r}}{\frac{\alpha}{2 \pi \lambda_{i z}}+\frac{\beta}{2 \pi \lambda_{g r}}} .
$$

Knowing the flow we can calculate the temperature of the soil at the place of the pipeline laying.

$$
t_{t}=t_{g}-\frac{\alpha q_{l}}{2 \pi \lambda_{i z}}
$$

A number of problems were solved based on the listed dependences.

\subsection{Determination of the soil temperature at the place of the underground pipeline laying}

We find the minimum temperature established in the process of freezing $t_{g r}$. To do it, we construct this graph ${ }_{n}^{t_{n}}(\tau)$ with a certain time step Fig. 1. At this point the minimum temperature of the soil is determined ${ }^{t_{g r}}$ related to the natural influence of the cold climate 
changes in the air temperature on the earth's surface. For Novy Urengoy we obtained the following variation of the soil temperature at the depth of the pipeline laying.

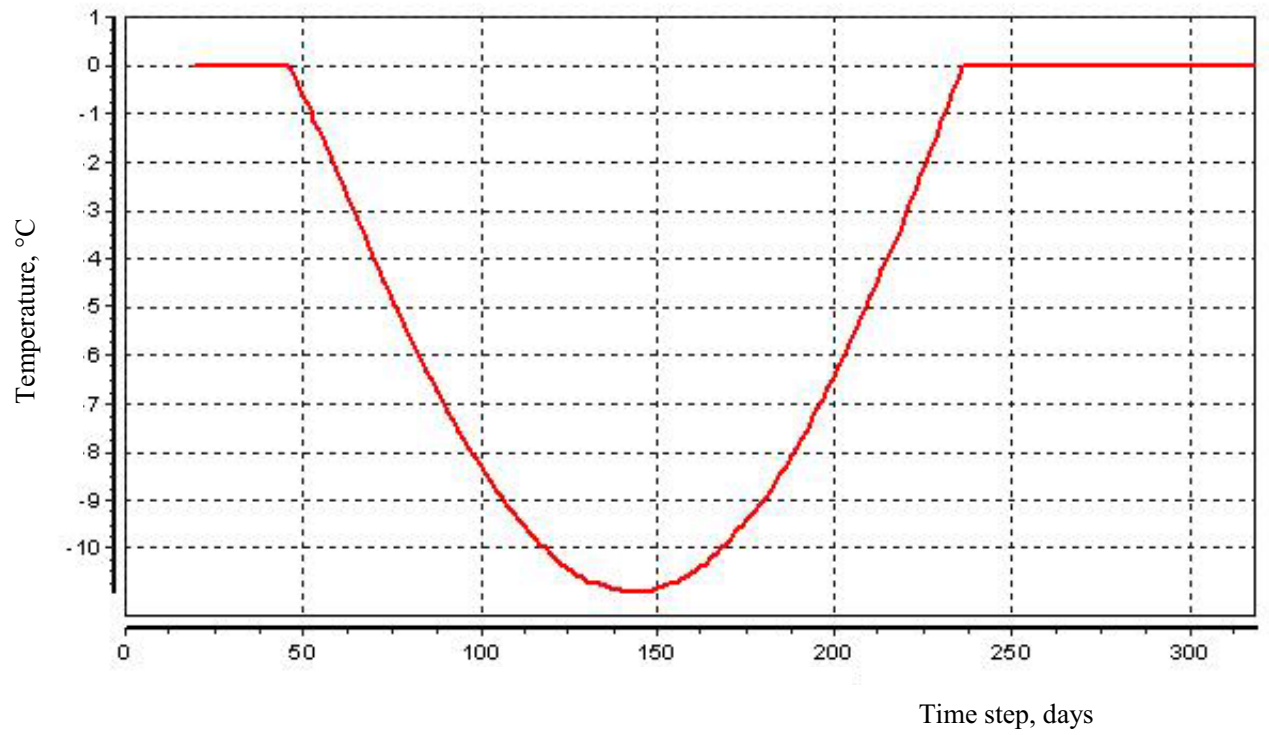

Fig. 1. Graph of the soil temperature dependence on time at a laying depth $-\mathrm{h}$

\section{2. Determination of the temperature on the pipe surface and the heat flow at a known insulation thickness}

Depending on the thickness of insulation ${ }^{{ }}$, which we specify, for example, by SNiP, using known values ${ }^{t_{g}}$ and ${ }^{t_{g r}}$, from the balance equation of heat flows we determine the flow from the warm pipe directed to the soil and the temperature on the surface of the heat conductor insulation. To do this, we find $\alpha, \beta$, determine the total resistance $R$ and:

$$
q_{l}=\frac{t_{g}-t_{g r}}{R}
$$

where:

$$
\begin{gathered}
R=\frac{\alpha}{2 \pi \lambda_{i z}}+\frac{\beta}{2 \pi \lambda_{g r}}, \\
\alpha=\ln \frac{D_{n}+2 \delta_{i z}}{D_{n}} ; \\
\beta=\ln \left(\frac{2 h}{D_{n}+2 \delta_{i z}}+\sqrt{\left(\frac{2 h}{D_{n}+2 \delta_{i z}}\right)^{2}-1}\right)
\end{gathered}
$$

The formula above determined by the geometrical dimensions of the pipe and the insulating layer. Thus, we can construct: 
- the dependence of flow $q_{l}$ on the laying depth in the frozen ground $h$ at a fixed insulation thickness $\delta_{i z}$.

- the soil temperature dependence at the depth of the pipeline.

Using the notation for the right side of the Forchheimer formula (the case of cylindrical symmetry):

$$
D=\exp \left(-\frac{t_{g r} \ln \left(\frac{2 h}{r}\right)}{\frac{\lambda_{t}}{\lambda_{m}} t_{t}-t_{g r}}\right),
$$

we rewrite the expression in a new form:

$$
\frac{y+\sqrt{h^{2}-r^{2}}}{y-\sqrt{h^{2}-r^{2}}}= \pm D .
$$

We solve this equation with respect to the distance from the surface of the soil to the beginning of the melt zone:

$$
y+\sqrt{h^{2}-r^{2}}= \pm D\left(y-\sqrt{h^{2}-r^{2}}\right) .
$$

For the variant with a plus we obtain:

$$
\begin{gathered}
y+\sqrt{h^{2}-r^{2}}=D\left(y-\sqrt{h^{2}-r^{2}}\right), y(1-D)=(-1-D) \sqrt{h^{2}-r^{2}}, \\
y=\frac{(-1-D) \sqrt{h^{2}-r^{2}}}{1-D},
\end{gathered}
$$

where $y$ - the distance to the lower edge of the melt zone.

For the variant with a minus:

$$
\begin{gathered}
y+\sqrt{h^{2}-r^{2}}=-D\left(y-\sqrt{h^{2}-r^{2}}\right), y(1+D)=(-1+D) \sqrt{h^{2}-r^{2}}, \\
y=\frac{(-1+D) \sqrt{h^{2}-r^{2}}}{1+D},
\end{gathered}
$$

where $y$ - the distance to the upper edge of the melt zone.

One of the calculation variants is shown in the graph (Fig. 2). 


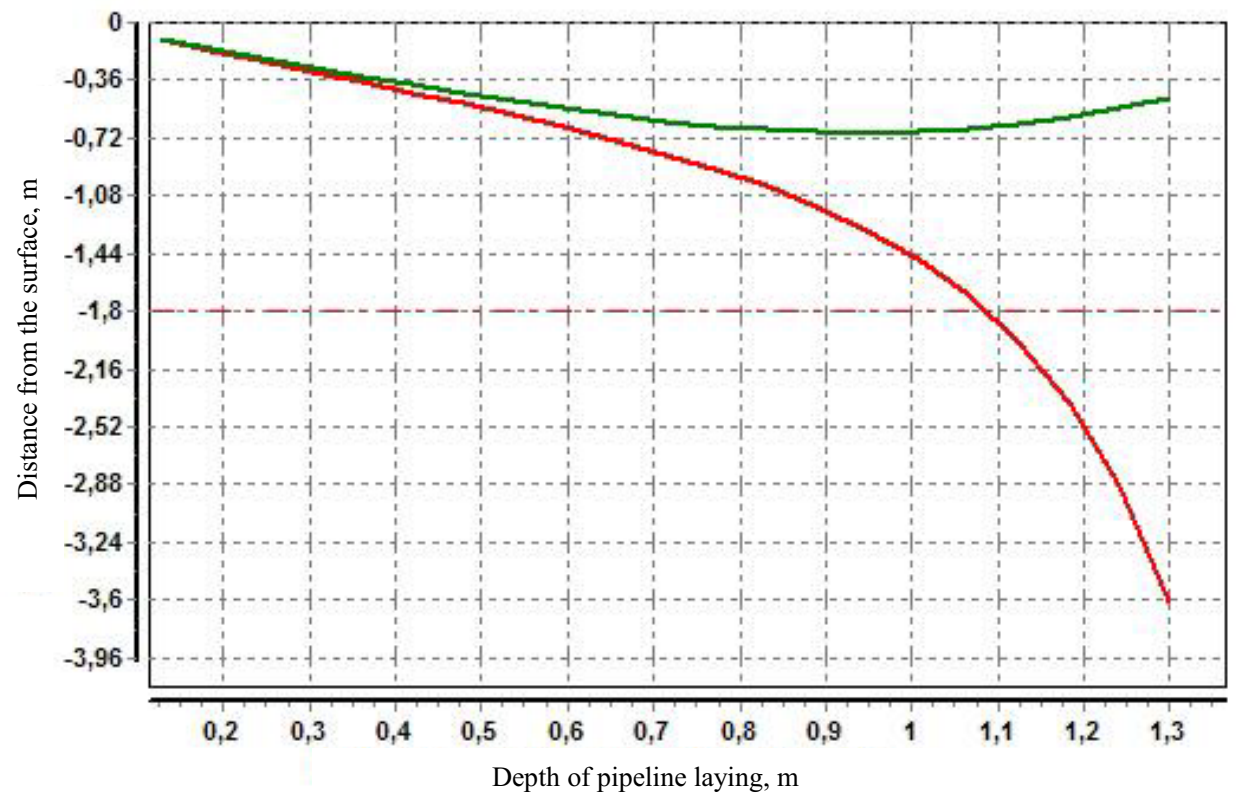

Fig. 2. Graph of thawing zones dependence on the laying depth

\section{Conclusions}

The authors studied the issues related to the peculiarities of pipeline construction in the north of Tyumen region, clarified the laws of formation of thawing halos around pipelines and determined the optimal conditions for laying pipelines.

According to the algorithm and the computer program, the authors made calculations for various options of the studied process with a change of parameters in a wide range of values. Figure 2 shows a variant of calculations, in which for the selected section (in this case $L_{c e}=2000 \mathrm{~m}$, total length $L=5000 \mathrm{~m}$ ) the dependence of the upper and lower limits of the melt zone on the laying depth of the pipeline is shown. For example, for the laying depth $\mathrm{h}=1$ meter the distance to the upper edge of the melt zone is equal to $y_{1}=0.7 \mathrm{~m}$, to the lower edge $y_{2}=1.4 \mathrm{~m}$ considering that the permafrost zone begins at the depth $H_{T}=1.8$ $\mathrm{m}$. Thus, by different signs of the right side of equation (19) it is possible to determine, respectively, the depth of the upper and lower edges of the thawing zone at different depths of laying. From the above calculations, the equilibrium temperatures of the soil and on the surface of the pipe were determined.

The research was carried out on the basis of a comprehensive program "Oil and gas in Western Siberia" [10].

\section{References}

1. S. D. Mordovskiy, E. E. Petrov, V. Yu. Izakson, Mathematical modeling of the twophase zone during the freezing-thawing of the permafrost (Nauka, Novosibirsk, 1997) 
2. B. V. Moiseev, Yu. D. Zemenkov, K. N. Ilyuhin, N. V. Nalobin, Higher Educational Institutions News. Oil and Gas, 4, 96-99 (2012)

3. N. M. Belyaev, A. A. Ryadko, Methods of nonstationary heat conduction (Vysshaya shkola, Moscow, 1978)

4. B. V. Moiseev, Yu. D. Zemenkov, S. M. Dudin, N. V. Nalobin, Territory Neftegaz Journal, 9, 112-117 (2015)

5. B. V. Moiseev, Yu. D. Zemenkov, N. V. Nalobin, Advances in current natural sciences, 8, 188-194 (2016)

6. B. V. Moiseev, A. D. Gerber, K. N. Ilyuhin, N. V. Nalobin, Calculation of temperature conditions of frozen soil around the pipeline (Rospatent, Moscow, 2016)

7. O. A. Stepanov, B. V. Moiseev, G. G. Hoperskiy, Heat supply at oil pumping stations (Nedra, Moscow, 1998)

8. V. E. Gubin, V. V. Gubin, Pipeline transportation of crude oil and petroleum products (Nedra, Moscow, 1992)

9. R. A. Aliev, V. D. Belousov, A. G. Nemudrov, Pipeline transportation of crude oil and gas (Nedra, Moscow, 1998)

10. Yu. D. Zemenkov, Operation of the main and technological oil and gas pipelines: textbook (TSOGU. Tymen, 2014) 